\title{
Prevalence of Plasmodium falciparum Infection (Malaria) Among Pregnant Women Attending Primary Health Care in Wushishi
}

\author{
Agholor Kin (Corresponding Author), Lucy F. Olusola, Idris Abubakar, Yakubu \\ Mahmud \\ Department of Biological Science, Niger state Polytechnic, Zungeru, Niger State, Nigeria. \\ E-mail: kinagholor@gmail.com, Phone No.: +2347062361650
}

Received: May 15, 2019 Accepted: July 1, 2019

doi:10.5296/jbls.v10i2.15344 URL: https://doi.org/10.5296/jbls.v10i2.15344

\begin{abstract}
Plasmodium falciparum is the leading cause of malaria in pregnant women, a disease of public health importance especially in Nigeria where the infection is endemic. Hence, this study was conducted to ascertain the prevalent rate of Plasmodium falciparum infection among pregnant women attending antenatal in primary health care center, Wushishi Local Government, Niger state, Nigeria. 150 pregnant women were randomly selected and tested for P. falciparum using Plasmodium falciparum (05FK50) Rapid Diagnostic Test kit. The result obtained, revealed that $36(24 \%)$ out of the 150 samples of the pregnant women were positive without any clinical manifestation of the infection. The result revealed that the prevalence rate was higher among women within 11-20 age group (43.8\%) than those within 21-30 (17.4\%) and 31 and above (9.0\%). It was also observed from the study that prevalence rate was lower among women who use Insecticide Treated Net (6.5\%) compared to those who does not $(52.6 \%)$. Therefore, the problem of Plasmodium falciparum infection in pregnant women should be prevented by the use of insecticide treated nets and effective case management with appropriate antimalarial drug during antenatal clinical visits.
\end{abstract}

Keywords: Malaria, Plasmodium falciparum, prevalence, pregnancy, RDT kit

\section{Introduction}

Plasmodium falciparum is the leading cause of Malaria, a disease of public health importance especially in Nigeria where the infection is endemic. Plasmodium falciparum is the most virulent and dominant malaria strain among other species (vivax, malariae, ovale and knowlesi). They are transmitted by the female anopheles' mosquito during a blood meal (Cottrell et al., 2015). The parasite lives in the erythrocytes of human blood (Muenworn et al., 
2009). Malaria infections have attracted global concerns. It is the most important parasitic disease that is endemic in Africa (Edwards et al., 2015). In this endemic area, malaria is a threat to both pregnant women and their babies as it accounts for about 2 million newborn deaths each year (WHO, 2003b). In Africa, Nigeria accounts for a quarter of all malaria cases in the 45 malaria-endemic countries (WHO, 2008). And about 140 million people live in areas of high malaria transmission (FMH, 2000).

The human protective anti-plasmodial activity is suppressed during pregnancy; this has a clinical consequence on pregnant women (Diallo et al., 2012). The symptoms of malaria in pregnant women vary with the level of immunity the pregnant women have acquired and the intensity of malaria transmission (WHO, 2003a). Malaria in pregnant women may result to spontaneous abortion, low birth weight, neonatal death, and intrauterine growth retardation (Cottrell et al., 2015). In some cases, malaria infection in pregnancy may lead to anaemia in the mother resulting to impairment of foetal nutrition and the presence of the parasites in the placenta, a leading cause of poor infant survival, development and health complications that can last well into adulthood (Dellicour et al., 2007).

The infection caused by the specie, $P$. falciparum during pregnancy is estimated to be the cause of 10,000 maternal deaths each year, $8 \%$ to $14 \%$ of all low birth weight babies and 75,000 to 200,000 of all infant deaths each year (Diallo et al., 2012). There is an estimated $25-30 \%$ of mortality in children under five years old and 300,000 deaths each year are due to malaria infection (Desai et al., 2015). The pregnant women, who are at high risk of the effects of the infection, need special protective measures to ensure their survival and improve birth outcome. However, antenatal clinic visits have been a key for the delivery of prevention package for pregnant women (Imwong et al., 2015). But studies have shown that $40 \%$ of pregnant women in African are present for the first time in antenatal clinics in the second trimester of their pregnancy (WHO, 2015).

The insecticide treated nets which is part of the prevention package delivered during the first antenatal clinic visit provide additional protection for the mother during the remaining trimesters of pregnancy and into the post-partum period (WHO, 2003b). Intermittent Preventive Treatment (IPT) with anti-malaria drugs is also made available as a routine part of antenatal care to pregnant women in their first and second pregnancies in highly endemic areas (WHO, 2003b). At present, sulfadoxine-pyrimethamine (SP) is given. IPT with at least two treatment doses of SP is highly effective in reducing the proportion of women with anaemia or placental malaria infection at delivery (McGready, 2012).

However, understanding the distribution of malaria is crucial for fighting the disease although some researchers have reported high prevalence of malaria in different parts of Nigeria (Uneke, 2008). This study was aimed at determining the prevalent rate of Plasmodium falciparum infection among pregnant women attending antenatal in the primary health care center at Wushishi Local Government, Niger state, Nigeria.

\section{Materials and Method}

\subsection{Materials}

The materials used in the research work includes: SD Bioline malaria Ag P.f (05FK50) RDT kit, cotton wool, methylated spirit, hand gloves, lancet and alcoholic swab 


\subsection{Method}

\subsubsection{Study Populations and Designs}

The study was conducted in Primary Health Care Wushishi, a rural area in Niger State. In this area, the report of malaria cases is usually high during the rainy season, which occurs between May and November. The women who were attending antenatal between within this period were randomly selected. Their bio-data and clinical data related to antenatal care visits, parity, bed net use and gestational age were obtained.

\subsubsection{Sample Collection (Sampling Technique)}

150 pregnant women at their different trimesters were selected randomly and verbally notified to seek their consent before sample collection.

\subsubsection{Methods of Sample Analysis}

RDT (Malaria antigen Pf/Pv, SD Bioline 05FK80, Europ Continents Ltd) was opened and labeled accordingly based on the patient identification number. The area of the hand of the patient to be lanced was cleaned with alcoholic swab and with the capillary pipette (5ul) provided, the blood sample was collected and dispensed into the round specimen well. Four drops of the assay diluent provided were placed into the square assay diluent well and allowed to stay for 15 minutes according to the manufacturer's instructions (SD Bioline 05FK80, Europ Continents Ltd). After which the test and the control bar was observed and the result was recorded based on the indications on the Kit.

\section{Result and Discussion}

\subsection{Result}

Table 1. Prevalence of Plasmodium falciparum base of the age of the pregnant women

\begin{tabular}{lccc}
\hline Age group & No. examined & No. infected & \% of infected women \\
\hline $11-20$ & 48 & 21 & 43.8 \\
$21-30$ & 69 & 12 & 17.4 \\
31 and above & 33 & 3 & 9.0 \\
Total & $\mathbf{1 5 0}$ & $\mathbf{3 6}$ & $\mathbf{2 4 . 0}$ \\
\hline
\end{tabular}

Among the 48 women examined within the age group of 10-20 years, 21 (43.8\%) were infected and 12 out of 69 women that were examined within the age of 21-30years were infected. However only 3 out of the 33 women examined within the age group of 33 years old and above tested positive thus, the women's young age and parity ( $<20$ years) could significantly be associated with the high prevalence rate as most of them are being pregnant for the first time. 
Table 2. Prevalence of Plasmodium falciparum based on gestational age of the pregnant women

\section{Gestational age}

T1 (1-12 weeks)

T2 (13- 24weeks)

T3 (25 - 36 weeks)

Total
No. examined

12

63

75

150
No. infected

0

24

12

16.0

24.0

From the above table, 12 women in their first trimester (1-12 weeks) were examined, and none of them were infected, the 63 women in their second trimester (13-24 weeks) that were examined, 24 (38.1\%) were infected, whereas those in their third trimester (25-36 weeks), 12 $(16.0 \%)$ out of the 75 women that were examined were infected.

Table 3. Prevalence of Plasmodium falciparum based on number of visit to antenatal care of the pregnant women

\begin{tabular}{cccc}
\hline Antenatal Care Visit & No. examined & No infected & \% of infected women \\
\hline $1^{\text {st }}$ & 57 & 21 & 36.8 \\
$2^{\text {nd }}$ & 33 & 6 & 18.2 \\
$3^{\text {rd }}$ & 27 & 6 & 22.2 \\
$\geq 4^{\text {th }}$ & 33 & 3 & 9.1 \\
Total $^{\text {th }}$ & $\mathbf{1 5 0}$ & $\mathbf{3 6}$ & $\mathbf{2 4 . 0}$ \\
\hline
\end{tabular}

The prevalence rate was higher in women who visited antenatal for the first time $(36.8 \%)$ compared to women $(9.1 \%)$ that visited $\geq$ four times.

Table 4. Prevalence of Plasmodium falciparum based on the use of insecticide-treated nets (ITNs)

\begin{tabular}{lccc}
\hline Bed net & No. Examined & No. infected & \% of infected women \\
\hline Used & 93 & 6 & 6.5 \\
Unused & 57 & 30 & 52.6 \\
Total & $\mathbf{1 5 0}$ & $\mathbf{3 6}$ & $\mathbf{2 4}$ \\
\hline
\end{tabular}


Out of the 150 women that was tested, 93 of them that uses ITN only $6(6.5 \%)$ was infected compared to 57 women that does not use ITN of which $30(52.6 \%)$ of them were infected. Thus, women who do not use a bed net (insecticide-treated nets) tended to have a higher risk of infection than those who slept under bed net (ITN).

\section{Discussion}

This study was carried out to assess the prevalence rate of malaria among pregnant women in Wushishi Local Government Area, Niger state, where malaria infection is endemic despite the distribution of both insecticides treated nets (ITNs) and the management of the infection with the antimalarial therapeutic drug, ACT in the area. In this present study, $36(24.0 \%)$ samples out of 150 samples of women attending antenatal care that were screened tested positive. This maternal prevalence rate of malaria (24.0\%) was higher compared to the study conducted in EBSUTH Facility in 2006 were the prevalence rate was (16\%), and in some other malaria endemic areas like the sub-Saharan Africa like Eastern Sudan where the prevalence of malaria in pregnant woman was 17.4\% (Kozuki, et al., 2013).

In relation to age, the prevalence was higher among women within 10-20-year-old (43.8\%) than those within 21-32 (17.4\%) and 33 and above (9.0\%). These could be as a result of the relationship between the parity and age of the women with malaria infection as reported by Walker et al., (2005) and WHO 2003b. Which was further explained by WHO (2002) that in the first and second pregnancies, women are more vulnerable to malarial infection caused by P. falciparum. Although most of the pregnant women sampled, did not show or complained of any signs and symptoms of malaria infection but their results were positive this indicates that the infection may not be clinically recognized unless otherwise diagnosed or investigated, which agrees with the work of Rijken et al., (2012) as one of the risk factors that pose danger to pregnant women living in malaria endemic area.

This study in relation to gestational age, showed that the prevalence of maternal malaria was higher among women in their second trimester $(38.1 \%)$ followed by those in their third trimester $(16.0 \%)$. This finding disagrees with the previous studies in other malaria endemic areas of sub-Saharan, where the level of malaria among pregnant women was significantly higher in their first then second trimester of gestation (Katz et al., 2013) but it agrees with the findings of Kozuki, et al., (2013), which they conducted in Bandiagara, Mali; and Eastern Sudan were prevalence rate of malaria infection was higher among pregnant women in their second and third trimester establishing that immunosuppression during the second trimester of pregnancy, as a result the presence of high adrenal steroid levels, as well as chorionic gonadotrophin and fetoprotein in the blood, may lead to depression of the lymphocyte activity, which may be responsible for higher susceptibility of women in their second and third trimester of pregnancy to the malaria parasite, as recorded in the studies conducted by Oliver et al; (2014).

In addition, there was decreased in the prevalence rate of malaria among the pregnant women from $36.8 \%$ to $9.1 \%$ with increased in antenatal visit from $1^{\text {st }}$ to $\geq 4^{\text {th }}$ visit. The relatively lower prevalence rates of malaria infection among pregnant woman who assess their antenatal care may not be attributed to acquired anti-malaria immunity only but could be as a 
result of increased malaria awareness among women in many endemic areas during their visit and interventions of various health authorities in the control and prevention of malaria in pregnancy (Jorgensen et al. 2010).

It was also observed that the prevalence rate of malaria among the pregnant women was lower in women who used Insecticide Treated Net (6.5\%) compared to women who do not (52.6\%). This agrees with the work of McGready et al., (2012) which shows that those who used Insecticide Treated Nets are less vulnerable to malaria infection (Muenworn et al., 2009). Therefore, women in high malaria transmission area should receive intermitted preventive treatment with an effective anti-malaria drug at regular scheduled Antenatal Care visits as recommended by WHO, (2003b).

\section{Conclusion}

The result of the finding revealed that $P$. falciparum infection is present among the pregnant women attending Primary Health Care Wushishi and the prevalence rate is significant $(24 \%)$. Therefore, more effort should be made towards the prevention of Plasmodium falciparum infection among pregnant women by creating more enlightenment on the need for early antenatal visit, the use of Insecticide Treated Nets and effective management of infected individual with appropriate antimalarial drug during antenatal clinical visits.

In addition, there should be effective malaria diagnosis for women during antenatal visit since both asymptomatic and symptomatic Plasmodium infection has deleterious consequences during pregnancy.

\section{References}

Cottrell, G., Moussiliou, A., Luty, A. J., Cot, M., Fievet, N., \& Massougbodji, A. (2015). Submicroscopic Plasmodium falciparum infections are associated with maternal anemia, premature births, and low birth weight. Clin Infect Dis., 60, 1481-1488. https://doi.org/10.1093/cid/civ122

Dellicour, S., Tatem, A. J., Guerra, C. A., Snow, R. W., \& Kuile, F. O. (2007). Quantifying the number of pregnancies at risk of malaria: a demographic study.

https://doi.org/10.1371/journal.pmed.1000221

Desai, M., Gutman, J., L'lanziva, A., Otieno, K., Juma, E., \& Kariuki, S, (2015). Intermittent screening and treatment or intermittent preventive treatment with dihydroartemisinin-piperaquine versus intermittent preventive treatment with sulfadoxine-pyrimethamine for the control of malaria during pregnancy in western Kenya: an open-label, three-group, randomized controlled superiority trial. Lancet, 386, 2507-2519. https://doi.org/10.1016/S0140-6736(15)00310-4

Diallo, A., Ndam, N. T., Moussiliou, A., Dos Santos, S., Ndonky, A., \& Borderon, M. (2012). Asymptomatic carriage of Plasmodium in urban Dakar: the risk of malaria should not be under estimated, 7, 3. https://doi.org/10.1371/journal.pone.0031100

Edwards, H. M., Canavati, S. E., Rang, C., Ly, P., Sovannaroth, S., \& Canier, L. (2015). 
Novel cross-borderapproaches to optimise identification of asymptomatic and artemisinin-resistant Plasmodium infection in mobile populations crossing Cambodian borders; 10, 124300. https://doi.org/10.1371/journal.pone.0124300

Federal Ministry of Health, (2000). Malaria situation analysis document, Nigeria: FederalMinistry of Health; P. 14.

Imwong, M., Nguyen, T. N., Tripura, R., Peto, T. J., Lee, S. J., \& Lwin, K. M. (2015). The epidemiology of subclinical malaria infections in South-East Asia: findings from cross-sectional surveys in Thailand-Myanmar border areas, Cambodia, and Vietnam. Malar J., 14, 381. https://doi.org/10.1186/s12936-015-0906-X

Jorgensen, P., Nambanya, S., Gopinath, D., Hongvanthong, B., Luangphengsouk, K., \& Bell, D. (2010). High heterogeneity in Plasmodium falciparum risk illustrates the need for detailed mapping to guide resource allocation: a new malaria risk map of the Lao People's Democratic Republic. Malaria J., 9, 59. https://doi.org/10.1186/1475-2875-9-59

Katz, J., Lee, A. C., Kozuki, N., Lawn, J. E., Cousen, S., \& Blencowe, H. (2013). Mortality risk in preterm and small-for-gestational-age infants in low-income and middle-income countries: A pooled country analysis. Lancet, 382, 417-425.

https://doi.org/10.1016/S0140-6736(13)60993-9

Kozuki, N., Lee, A. C., Silveira, M. F., Sania, A., Vogel, J. P., \& Adair, L. (2013). The associations of parity and maternal age with small-for-gestational-age, preterm, and neonatal and infant mortality: a meta-analysis. BMC Publ Health., 13(Suppl 3), S2.

https://doi.org/10.1186/1471-2458-13-S3-S2

McGready, R., Lee, S. J., Wiladphaingern, J., Ashley, E. A., Rijken, M. J., \& Boel, M. (2012). Adverse effects of falciparum and vivax malaria and the safety of antimalarial treatment in early pregnancy: a population-based study. Lancet Infect Dis., 12, 388-396.

https://doi.org/10.1016/S1473-3099(11)70339-5

Muenworn, V., Sungvornyothin, S., Kongmee, M., Polsomboon, S., \& Bangs, M. (2009). Bitingactivity and host preference of the malaria vectors Anopheles maculatus and Anophelessawadwongporni (Diptera: Culicidae) in Thailand. J. Vector Ecol., 34, 62-69. https://doi.org/10.1186/1756-3305-5-211

Oliver, O., Angela, I., Nneka, L. O., \& Ngozi, O. (2014). Prevalence of malaria among pregnant mothers and possible relationship to parity in Abakaliki, Southeast Nigeria. European Journal of Experimental Biology, 4(4), 15-19.

https://doi.org/10.18535/jmscr/v6i12.13

Rijken, M., Papageorghiou, A., Thiptharakun, S., Kiricharoen, S., Dwell, S. L., \& Wiladphaingern, J. (2012). Ultrasound evidence of early fetal growth restriction after maternal malaria infection; 7, e31411. https://doi.org/10.1371/journal.pone.0031411

Uneke, C. J. (2008). Assessment of malaria in pregnancy using rapid diagnostic tests and its association with HIV infection and haematologic parameters in south-eastern Nigeria. 


\section{Macrothink}

Haematologica, 93, 143-144. https://doi.org/10.3324/haematol.11695

Walker, A., Djokam, R. R., Eno, A., Leke, R. F., Titanji, V. P., \& Fogako, J. (2005). Malaria inpregnant Cameroonian women: the effect of age and gravidity on submicroscopic and mixed species infections and multiple parasite genotypes. Am J Trop Med Hyg., 72, 29-35. https://doi.org/10.4269/ajtmh.2005.72.229

WHO (2015). Global Malaria Programme. World Malaria Report, Geneva: World Health Organization; http://www.who.int/malaria/publications/world-malaria-report2015/report/en/

World Health Organization (2003a). Strategic Framework for Malaria Control during Pregnancy in the WHO Africa Region. Geneva, WHO

World Health Organization (2003b). Antenatal Care in Developing Countries. Promises, Achievements and Missed Opportunities. An Analysis of levels, Trend and Differentials 1990-2001.

World Health Organization, (2008). World Malaria Report Switzerland: World HealthOrganization; P. 99-101.

\section{Copyright Disclaimer}

Copyright for this article is retained by the author(s), with first publication rights granted to the journal.

This is an open-access article distributed under the terms and conditions of the Creative Commons Attribution license (http://creativecommons.org/licenses/by/3.0/). 